\title{
Connectivity Changes Underlying Spectral EEG Changes during Propofol-Induced Loss of Consciousness
}

\author{
Mélanie Boly, ${ }^{1,2}$ Rosalyn Moran, ${ }^{2}$ Michael Murphy, ${ }^{3}$ Pierre Boveroux, ${ }^{1}$ Marie-Aurélie Bruno, ${ }^{1}$ Quentin Noirhomme, ${ }^{1}$ \\ Didier Ledoux, ${ }^{1,4}$ Vincent Bonhomme, ${ }^{4}$ Jean-François Brichant, ${ }^{4}$ Giulio Tononi, ${ }^{3}$ Steven Laureys, ${ }^{1}$ and Karl Friston ${ }^{2}$ \\ ${ }^{1}$ Coma Science Group, Cyclotron Research Centre and Neurology Department, University of Liège and Sart Tilman Hospital, 4000 Liège, Belgium, \\ ${ }^{2}$ Wellcome Trust Centre for Neuroimaging, Institute of Neurology, University College London, London WC1N 3BG, United Kingdom, ${ }^{3}$ School of Medicine, \\ Department of Psychiatry, University of Wisconsin, Madison, Wisconsin 53719, and ${ }^{4}$ Anesthesiology and Intensive Care Department, University of Liège \\ and Centre Hospitalier Universitaire Sart Tilman Hospital, 4000 Liège, Belgium
}

The mechanisms underlying anesthesia-induced loss of consciousness remain a matter of debate. Recent electrophysiological reports suggest that while initial propofol infusion provokes an increase in fast rhythms (from beta to gamma range), slow activity (from delta to alpha range) rises selectively during loss of consciousness. Dynamic causal modeling was used to investigate the neural mechanisms mediating these changes in spectral power in humans. We analyzed source-reconstructed data from frontal and parietal cortices during normal wakefulness, propofol-induced mild sedation, and loss of consciousness. Bayesian model selection revealed that the best model for explaining spectral changes across the three states involved changes in corticothalamic interactions. Compared with wakefulness, mild sedation was accounted for by an increase in thalamic excitability, which did not further increase during loss of consciousness. In contrast, loss of consciousness per se was accompanied by a decrease in backward corticocortical connectivity from frontal to parietal cortices, while thalamocortical connectivity remained unchanged. These results emphasize the importance of recurrent corticocortical communication in the maintenance of consciousness and suggest a direct effect of propofol on cortical dynamics.

\section{Introduction}

The mechanisms underlying anesthesia-induced loss of consciousness (LOC) are unknown. Previous neuroimaging studies performed during anesthesia have shown a breakdown of connectivity in frontoparietal networks (Lee et al., 2009; Boveroux et al., 2010). Whether this decreased connectivity is due to a direct cortical action of anesthetics, or to a disruption of corticothalamocortical connectivity, remains unclear (Boveroux et al., 2010). Some authors suggest that anesthetics may act by depressing thalamic function, impairing its role either as an arousal promoter or as a cortical read-out (Alkire et al., 2008). Many studies have reported a reduction of thalamic metabolism and blood flow during anesthesia (Fiset et al., 1999; Alkire and Miller, 2005; Alkire et al., 2008). However, during the first few minutes of propofol-induced LOC, thalamic activity seems to be preserved, while cortical activity is depressed (Velly et al., 2007). It has also

\footnotetext{
Received July 21, 2011; revised March 19, 2012; accepted March 26, 2012.

Author contributions: M.B., P.B., Q.N., V.B., J.-F.B., G.T., and S.L. designed research; M.B., P.B., M.-A.B., Q.N., and D.L. performed research; M.B., R.M., M.M., and K.F. analyzed data; M.B., R.M., M.M., P.B., M.-A.B., Q.N., V.B., J.-F.B., G.T., S.L., and K.F. wrote the paper.

This work was supported by the Belgian Fonds National de la Recherche Scientifique (FNRS), European Commission (Mindbridge, DISCOS, CATIA, and DECODER), Mind Science Foundation, James MCDonnell Foundation, French Speaking Community Concerted Research Action (ARC 06/11-340), Fondation Médicale Reine Elisabeth, Fonds Léon Frédéricq, and the National Institutes of Health. M.B., MA.B., and Q.N. are Postdoctoral Fellows, and S.L. is Senior Research Associate at the FNRS. R.M. and K.F. are supported by the Wellcome Trust. G.T. is supported by NIH.

The authors declare no competing financial interest.

Correspondence should be addressed to Mélanie Boly, Cyclotron Research Centre, Allée du 6 août, B30, 4000 Liège, Belgium. E-mail:mboly@ulg.ac.be.

DOI:10.1523/JNEUROSCI.3769-11.2012

Copyright $\odot 2012$ the authors $\quad 0270-6474 / 12 / 327082-09 \$ 15.00 / 0$
}

been suggested that anesthetic effects on the thalamus may be largely indirect (Alkire et al., 2000; Schiff and Plum, 2000; Franks, 2008). Indeed, the metabolic and electrophysiological effects of anesthetics on the thalamus in animals are abolished by removal of the cortex (Nakakimura et al., 1988; Angel, 1993; Vahle-Hinz et al., 2007). In short, the thalamus versus cortex debate remains active in the anesthesia literature (Alkire et al., 2008).

A recent study investigated EEG correlates of propofolinduced LOC in man (Murphy et al., 2011). Mild sedation (with a slower response to command but preserved consciousness) was characterized by a rise in high-frequency power in scalp EEG. Conversely, LOC was paired with the appearance of highamplitude delta waves on a persistent fast rhythmic background (Murphy et al., 2011). Furthermore, the same default network areas-i.e., precuneus/posterior cingulate cortex (PCC) and medial prefrontal/anterior cingulate cortex (ACC) - appeared to support both traveling slow waves and high-frequency EEG power increases. This observation is puzzling, because it is thought that the generation of fast and slow frequencies is mediated by opposing cellular mechanisms (Steriade, 2006): fast rhythms are produced when neurons are in a depolarized "awake" state, while slow rhythms are generated when neurons are hyperpolarized. The simultaneous presence of both activity modes in the same EEG (as well as their distinct profile of emergence across levels of sedation) therefore suggests distinct mechanisms in their generation.

The present study investigated the neural mechanisms underlying EEG spectral power changes observed during propofolinduced unconsciousness. This inquiry has been enabled by 


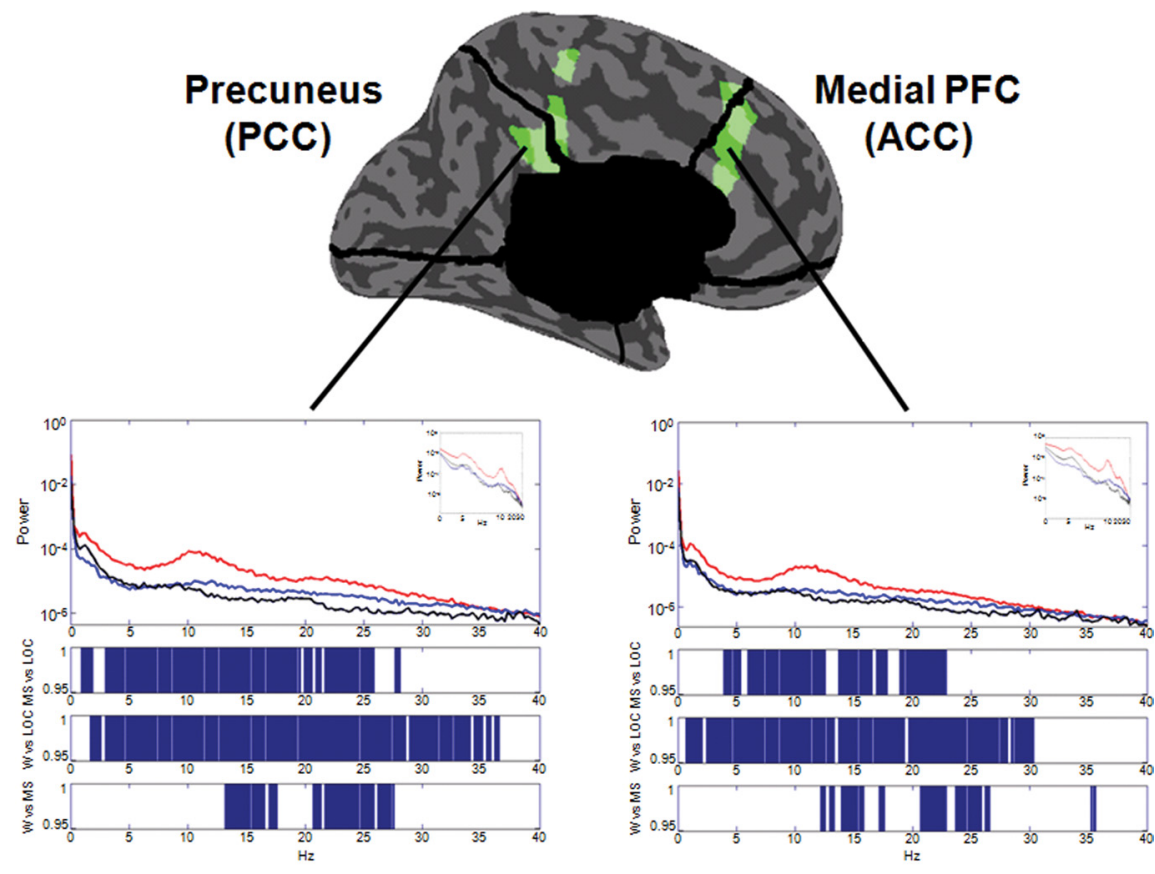

Cross-spectral density

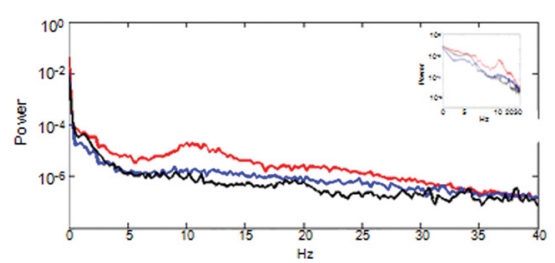

Figure 1. Power spectrum changes across vigilance states in two sources showing increased gamma power in $\mathrm{LOC}$, compared with wakefulness ( $p<0.05$, corrected using SnPM). The frontal source or ROI is a portion of the anterior cingulate, and the posterior ROI is a portion of the posterior cingulate. Semi-logarithmic plots of power spectra from each region and cross-spectral density between the two regions are displayed in waking (W, black), mild sedation (MS, blue), and LOC (red); the insets are the corresponding log-log plots. The blue bars in the middle panel show frequency band-specific changes in power during the transition from waking to sedation and then to LOC (when considered significant at $p<0.05$, corrected using SnPM).

recent advances in the analysis of EEG data, specifically, dynamic causal modeling (DCM) (Friston et al., 2003). DCM uses a biologically informed generative model that embodies known constraints on the generation of EEG signals (Kiebel et al., 2009). Bayesian model inversion and comparison then provide a method for testing candidate models of causal interactions among brain sources in a distributed network. In particular, DCM for steady-state responses (SSRs) allows one to explain neural mechanisms underlying the differences in spontaneous EEG power spectra (Moran et al., 2009) and was used here to quantify changes in neural coupling during propofol-induced unconsciousness.

\section{Materials and Methods}

Data acquisition. The data used in this study have been reported previously by Murphy et al. (2011) in an analysis of functional connectivity using coherence measures. In brief, the study was approved by the Ethics Committee of the Faculty of Medicine of the University of Liège and written informed consent was obtained from all participants. None of the subjects had a history of head trauma or surgery, mental illness, drug addiction, asthma, motion sickness, or previous problems during anesthesia. Fifteen minute spontaneous 256-electrode, high-density EEG recordings were acquired in eight subjects (mean age $22 \pm 2$ years, 4 males) in four different states: normal wakefulness, sedation (slower response to command, Ramsay scale score 3), LOC with clinical unconsciousness (no response to command, Ramsay scale score 5), and recovery of conscious- ness (Ramsay et al., 1974). Subjects were considered fully awake or to have recovered consciousness if the response to verbal command ("squeeze my hand") was clear and strong (Ramsay 2), in sedation if the response to verbal command was clear but slow (Ramsay 3 ), and in LOC if there was no response (Ramsay 5-6). For each consciousness level assessment, Ramsay scale verbal commands were repeated twice. The most comfortable supine position was used to avoid painful stimulation related to position. All recordings were performed with eyes closed.

Propofol was infused through an intravenous catheter placed into a vein of the right hand or forearm. Throughout the study, the subjects breathed spontaneously and additional oxygen $(5 \mathrm{~L} / \mathrm{min})$ was given through a loosely fitting plastic facemask. Computercontrolled intravenous infusion of propofol (total intravenous anesthesia; Alaris) was used to obtain constant effect-site concentrations. The propofol plasma and effect-site concentrations were estimated using a threecompartment pharmacokinetic model (Marsh et al., 1991). Average arterial blood concentrations of propofol were $1.91 \pm 0.52 \mu \mathrm{g} / \mathrm{ml}$ for sedation and $3.87 \pm 1.39 \mu \mathrm{g} / \mathrm{ml}$ for LOC.

Data preprocessing. Thirty second artifactfree EEG epochs from each condition were selected for analysis. The raw signals were filtered from 0.5 to $40 \mathrm{~Hz}$. A standard set of electrodes was coregistered to the magnetic resonance image of an individual whose head closely approximates the Montreal Neurological Institute (MNI) space. The electromagnetic forward model comprised four nested spheres. The inverse matrix was calculated using a constrained, depth- and orientation-weighted, truncated singular value decomposition regularized $\left(10^{-3}\right)$ L2 minimum norm (Grave de Peralta Menendez et al., 2001). The source space was a set of 2447 cortical voxels $\left(7 \mathrm{~mm}^{3}\right)$ that were identified based on the MNI probabilistic brain atlas. The source space was restricted to the cortex because cortical pyramidal neurons are the most likely generators of the EEG (Kirschstein and Köhling, 2009). For the power analysis, we source localized about $5 \mathrm{~min}$ of artifact-free EEG from each subject in each condition. Source modeling was performed in GeoSource (Electrical Geodesics), while all other calculations were performed in MATLAB (MathWorks). From this source reconstruction, we selected two regions of interest (ROIs) based on a previous study showing increased gamma power during LOC compared with wakefulness (Murphy et al., 2011). Figure 1 shows group mean source-level power spectra and cross-spectral densities (in semi-log and log-log scales) from the frontal and parietal ROIs during wakefulness, sedation, and LOC. Statistical nonparametric mapping $(\mathrm{SnPM})$ was used to compare frequency spectra between behavioral states (Nichols and Holmes, 2002). Power spectrum change statistics were performed on a $1 \times 1 \mathrm{~Hz}$ frequency bin basis and were run in MATLAB (MathWorks) using SnPM - a permutation-based image analysis scheme that accounts for multiple comparisons (Nichols and Holmes, 2002).

Extraction of frontal and parietal source activity. The rationale of the present work was to investigate changes in a frontoparietal network that caused changes in the power spectrum observed during two levels of propofol sedation. In DCM, this is modeled explicitly in terms of neuronal subpopulations that influence each other through intrinsic (withinarea) and extrinsic (between-area) connections. The parameters of these models control the expression of synaptic responses resulting from these 
influences. Model inversion allows us to estimate these parameters and evaluate the evidence for one model or network architecture, relative to others. In brief, we extracted the source-reconstructed signals from two sources or regions of interest, previously determined to be the major contributors to increases in gamma power $(25-40 \mathrm{~Hz})$ during propofolinduced unconsciousness (see Data preprocessing, above). Increases in gamma power during loss of consciousness are particularly interesting, given that gamma frequency activity is thought to be important for consciousness (Llinás et al., 1998). Source-reconstructed signals were modeled with DCM to characterize the mechanisms underlying changes in power spectra across the three states. Bayesian model selection (BMS) compared the evidence of a model with exclusively cortical sources to that of two other thalamocortical models. These thalamocortical models comprised one or two thalamic sources connected reciprocally with the cortical sources. Having established the best model, we then quantified the changes in neural excitability and connectivity with sedation and LOC.

For DCM, we summarized the activity of each region (frontal and parietal) using its principle eigenvariate. The first eigenvariate of frontal and parietal activity was calculated from 9 dipolar sources (vertices) per region, over a $30 \mathrm{~s}$ period in each condition. These were the time series used for subsequent dynamic causal modeling: DCM uses an in-built AR process to compute a regularized frequency domain representation (found in the spectral toolbox of SPM8, http://www.fil.ion.ucl.ac.uk) from the time series. We evaluated three DCMs for each subject that explained differences among the conditions (wakefulness, sedation, and LOC) in terms of changes in intrinsic and extrinsic coupling.

Network models or hypotheses. DCM is usually used in a hypothesisdriven fashion: it does not explore all possible models but tests specific mechanistic hypotheses (Boly et al., 2011). Here, we examined three models, corresponding to hypotheses about the neural mechanisms underlying the spectral changes observed during propofol-induced loss of consciousness (Fig. 2A). Our first model comprised two cortical areas, connected by forward and backward connections. A second model incorporated a (hidden) thalamic source, reciprocally connected to both cortical areas (i.e., no data were reconstructed in this region). The third model used two thalamic modules, with a similar architecture, but separately connected to each cortical source.

Dynamic causal modeling. The idea behind DCM is that M/EEG data are the response of a dynamic input-output system to endogenous fluctuations or exogenous (experimental) inputs. One models the measured $\mathrm{M} / \mathrm{EEG}$ as the response of a network of sources, where each source corresponds to a neural mass model of several subpopulations. In DCM for steady-state responses, neuronal dynamics are assumed to be driven by endogenous (neuronal) fluctuations with an unknown but parameterized spectral density. This sort of DCM is useful, for example, in sleep research, where there are no external or exogenous stimuli (Moran et al., 2009). We employ these modeling assumptions here, assuming the endogenous neuronal fluctuations were a mixture of pink $(1 / f)$ and white noise.

Assuming the system operates in a steady state around its fixed point, we can linearize the nonlinear differential equations (Fig. 2B) describing its response in the frequency domain. These differential equations depend on the neural mass model of sources in the network and the effective connectivity or coupling among them. This linearization allows one to compute the transfer function mapping from the endogenous (neuronal) fluctuations to the sensors, where the transfer functions are the Fourier transforms of the source's kernels (Fig. 2 B). Applying this transfer function to the spectral density of these inputs provides the predicted spectral activity in each region. This allows us to establish a mapping from the system parameters to the predicted frequency spectrum (Moran et al., 2007). Furthermore, we can model differences between two or more spectra, acquired under different conditions, as a consequence of changes in parameters. These parameters correspond here, to intrinsic or extrinsic connections, where intrinsic connections are controlled by the maximum amplitude of postsynaptic potentials (Kiebel et al., 2007). The basic idea is to manipulate the (real) system (e.g., by changing levels of propofol sedation) and model the experimental effects in terms of changes in coupling. Estimated parameter changes across the three levels of consciousness served as summary statistics in classical tests (ANOVA and post hoc $t$ tests) of condition-specific effects over subjects.

In detail, we used a neural mass model for each source, which describes the dynamics of three neuronal subpopulations. Each population has its own (intrinsic) dynamics governed by the neural mass equations (Moran et al., 2009), but also receives endogenous input from surrounding cortex or from other sources. The sources and their interactions are fully specified by first-order differential equations that are formally related to neural mass models used in computational models of M/EEG (Breakspear et al., 2006; Rodrigues et al., 2010). These differential equations describe the evolution of hidden neuronal states (membrane potentials and currents) in the subpopulations comprising each source. For the cortex, these subpopulations are layer specific, with spiny stellate cells in the granular layer that are reciprocally connected to pyramidal cells in infragranular and supragranular layers. Inhibitory interneurons in the infragranular and supragranular layers are, in turn, reciprocally connected to the pyramidal cells. Connections between regions originate and terminate at specific cortical layers. In this way, DCM for SSRs allows one to specify hierarchical neuronal networks with anatomically grounded connectivity rules (Felleman and Van Essen, 1991). Prefrontal and parietal cortices are thought to be hierarchically arranged with laminar specific bidirectional coupling (Jacobson and Trojanowski, 1977; Schwartz and GoldmanRakic, 1984). Generally, forward connections originate in superficial pyramidal cells (in supragranular layers II and III) and terminate in the granular layer IV (Pandya, 1995; Rouiller and Durif, 2004). In contrast, reciprocal backward connections arise from the deep pyramidal cells (in (infragranular layers V and VI) and terminate outside of layer IV (Winguth and Winer, 1986; Rouiller and Durif, 2004; Moran et al., 2009). While our model lumps together supragranular and infragranular pyramidal cells, it accommodates these asymmetries by retaining their major functional characteristics; namely, forward connections arise in pyramidal cells and target spiny stellate cells in layer VI, whereas backward connections target pyramidal and inhibitory cells in supragranular and infragranular layers. The thalamic architecture was based on the model used by Robinson et al. (2001) and Breakspear et al. (2006) in previous characterizations of EEG spectra and seizure activity: thalamic sources comprised excitatory (relay cells) and inhibitory (reticular cells) subpopulations that were reciprocally connected. The thalamus sent forward connections to both cortical areas and received backward connections. We assume that the depolarization of pyramidal cell populations generates observed M/EEG data. In our case (because we used source reconstructed activity), there was a direct mapping between the activity of pyramidal cells and the virtual sensor in the two cortical sources.

Bayesian model inversion and estimation. Model inversion means fitting the model to the empirical data; here the cross-spectral densities from the cortical sources. In DCM a Variational Bayesian scheme called Variational Laplace (Friston et al., 2007) is used to approximate the conditional or posterior density $q(\theta)$, over parameters by maximizing the negative free energy:

$$
F=\ln p(y \mid m)-K L[q(\theta), p(\theta \mid y, m)] .
$$

Here $\mathrm{KL}$ is the divergence between the true $p \theta \mid y, m$ and approximate $q(\theta)$ posteriors. It can be seen from Equation 1 that the negative free energy is also a lower bound on the $\log$ model evidence, $\ln p(y \mid m)$ (the probability of the data $y$ given a model $m$ ) (for details, see Friston and Stephan, 2007). This quantity is used for model selection when testing a series of candidate architectures; in this case, a model with cortical sources was compared with two thalamocortical models. A random effects BMS analysis was performed (Stephan et al., 2009) to select the best model. The maximum a posteriori (or conditional mean) of the parameters encoding changes in connectivity then serve to quantify differences among experimental conditions. We modeled the three condition-specific effects, corresponding to wakefulness, sedation, and LOC, in each individual DCM. Effects were modeled in terms of changes (relative to the first condition) in the intrinsic connectivity (excitability) in all sources and the extrinsic connectivity between sources. To assess the consistency and significance of these effects over subjects, subject-specific changes were entered into an ANOVA with anesthetic depth as a within-subject factor. Two-tailed 
A

Model 1
P

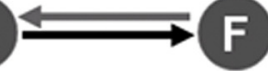

Model 2

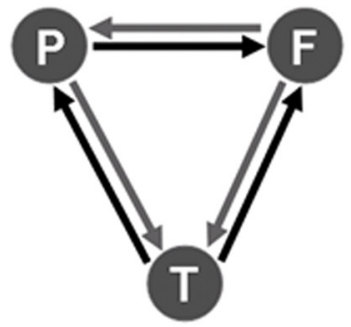

Model 3

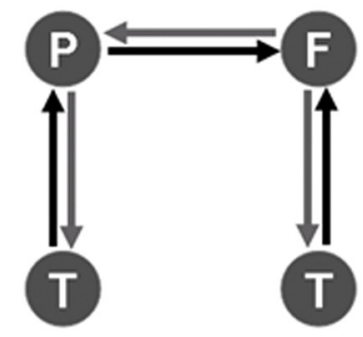

B

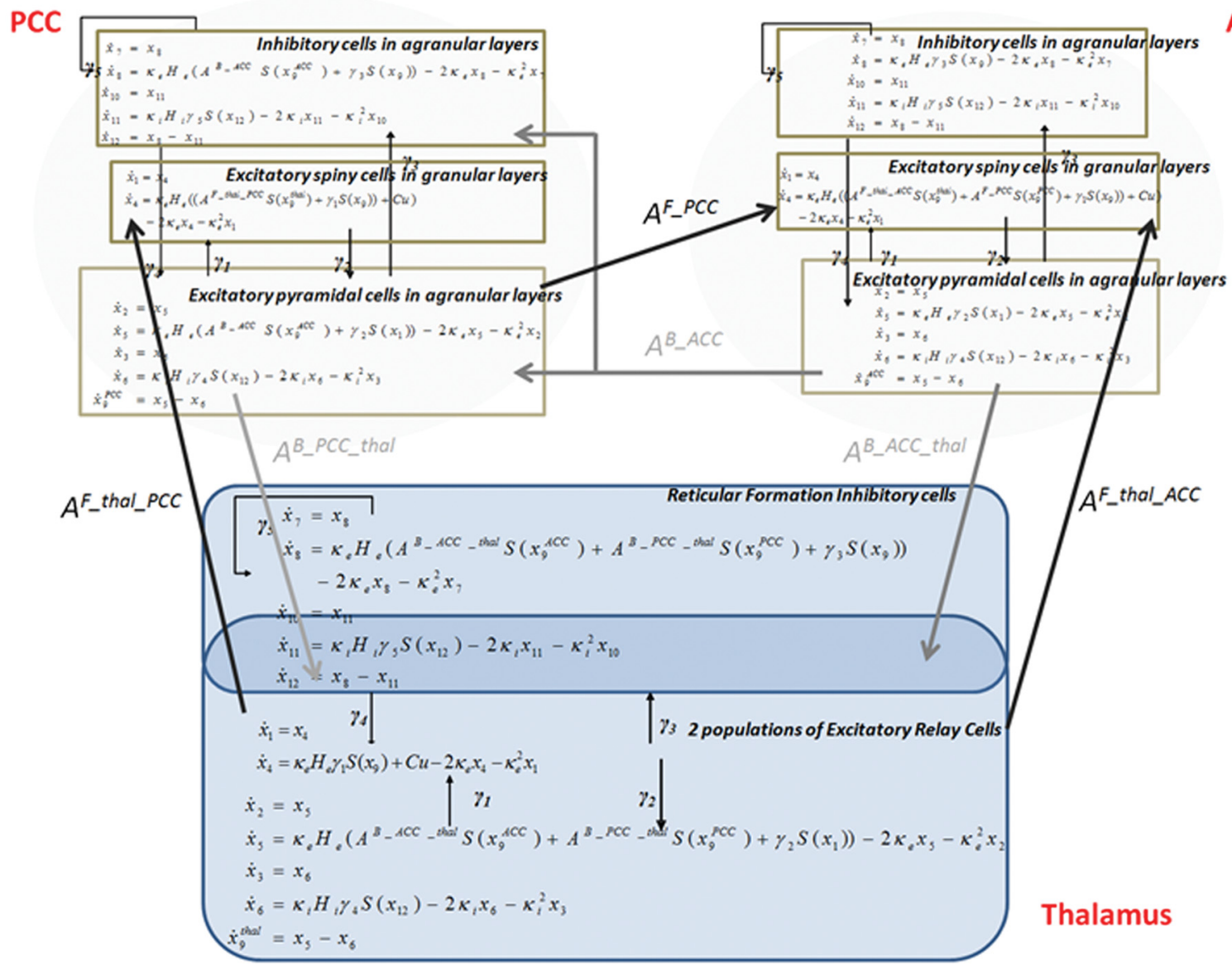

Figure 2. A, Model specification: the three DCMs used for Bayesian model comparison. Sources in each model receive endogenous neuronal fluctuations or innovations (modeled as a mixture of pink and white noise) that elicitneural responses characterized by a power spectrum that depends upon the model parameters. Random-effects Bayesian model selection showed that the model with a common thalamic source (Model 2) had the greatest evidence (with a $92 \%$ posterior probability). Model 2 was thus selected for subsequent quantitative analysis of effective connectivity changes across the three experimental conditions. $\boldsymbol{B}$, Equations of motion describing neuronal interactions in thalamic, posterior cingulate, and anterior cingulate regions. These dynamics are based on a neural mass model that has been used extensively in the causal modeling of electromagnetic data (Jansen and Rit, 1995; David and Friston, 2003; David et al., 2005; Moran et al., 2008). It represents a minimal description of synaptic processing in multiple populations, in terms of synaptic convolution of presynaptic inputs and a nonlinear mapping between the resulting depolarization and firing rates. The kernels, from which we derive the transfer functions, obtain analytically from the Jacobian $\mathfrak{I}=\partial f / \partial x$ describing the stability of motion $\dot{x}=f(x, u, \theta)$ of hidden neuronal states, $x(t)$ and a mapping (forward model) $s(x, \theta: x \rightarrow s$ that couples hidden states to the observed signals in PCC and ACC. Neuronal fluctuations or input are denoted by $u(t)$. For region $i$, and input or innovation $k$, the kernel is:

$$
\begin{aligned}
K_{i}^{k}(\tau, \theta) & =\frac{\partial s_{i}(t)}{\partial u_{k}(t-\tau)} \\
& =\frac{\partial s_{i}(t)}{\partial g(t)} \frac{\partial g(t)}{\partial x(t)} \frac{\partial x(t)}{\partial x(t-\tau)} \frac{\partial x(t-\tau)}{\partial \dot{x}(t-\tau)} \frac{\partial \dot{x}(t-\tau)}{\partial u_{k}(t-\tau)} \\
& =\frac{\partial g_{i}}{\partial x} \exp (\mathfrak{I} \tau) \mathfrak{I}^{-1} \frac{\partial f}{\partial u_{k}}
\end{aligned}
$$

The Jacobian is augmented by a matrix of inter-regional delays $D, \mathfrak{I} \leftarrow(I+D \mathfrak{I})^{-1} \mathfrak{I}$. The kernels are hence functions of the model's equations of motion and output mapping. The output here is determined largely by pyramidal cell activity $(\sim 80 \%)$ in each cortical area. See Tables 1 and 2 in Moran et al. $(2009)$ for the prior values of model parameters. 


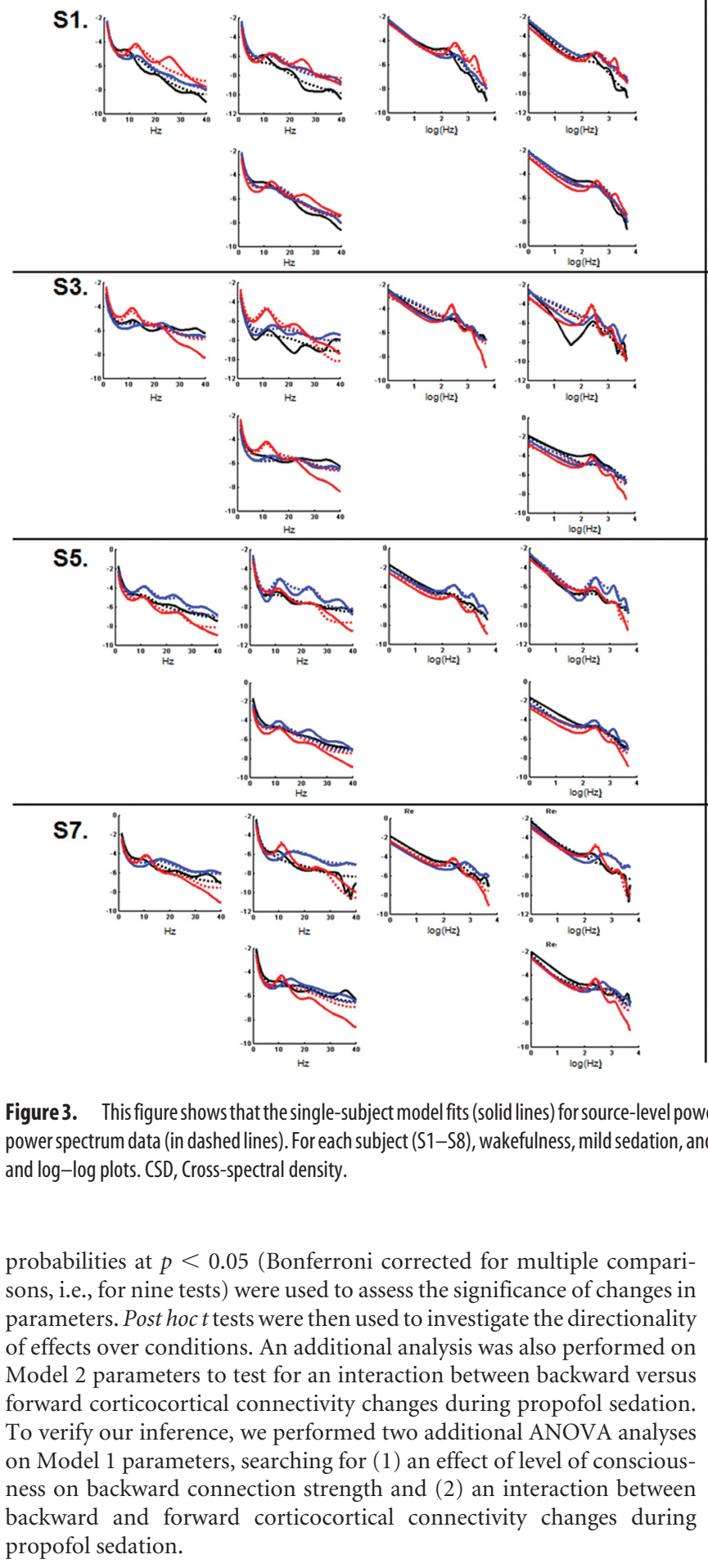

\section{Results}

The two regions of interest we investigated were located in precuneus/posterior cingulate and in medial prefrontal cortex. While initial propofol infusion was accompanied by an increase in fast frequencies in the beta-gamma range, both regions showed a further significant increase in slow-frequency power (delta to alpha range) with the emergence of clinical unconsciousness (Fig. 1). To characterize the neuronal mechanisms underlying these changes in power spectrum, we extracted the time-series of these two regions during wakefulness, mild sedation, and LOC, and modeled them with DCM under steady-state assumptions. We inverted three models for each subject (Fig. and $\log -\log$ plots. CSD, Cross-spectral density.
S2.
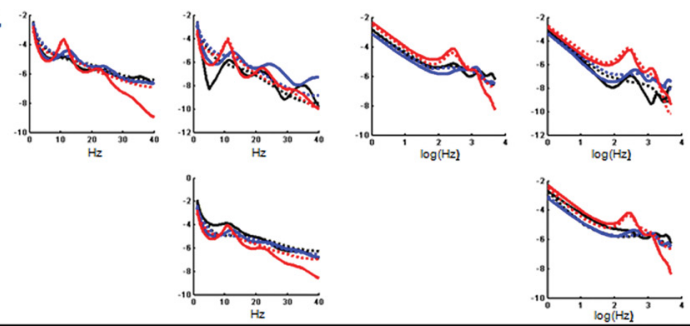

S4.
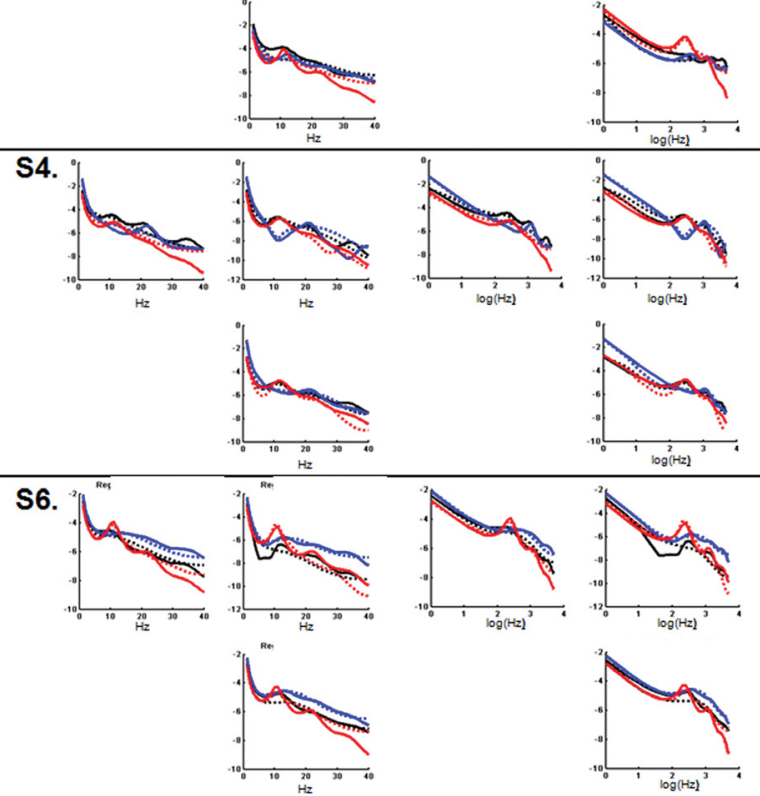

s8.
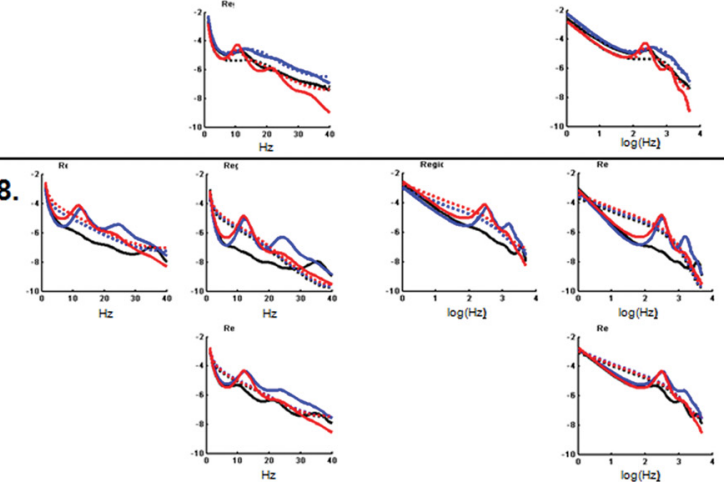

Figure 3. This figure shows that the single-subject model fits (solid lines) for source-level power spectra and cross-spectral densities as computed by DCM could satisfyingly account for the original

$2 A$ ). Model 1 comprised the two cortical areas that were connected reciprocally by backward and forward extrinsic connections. In Model 2, a thalamic source was added (Robinson et al., 2001; Breakspear et al., 2006), connected reciprocally to both cortical areas by forward (thalamocortical) and backward (corticothalamic) connections (Fig. $2 \mathrm{~B}$ ). Model 3 comprised the two cortical areas, each connected reciprocally with its own thalamic region. Bayesian model comparison revealed that the best model for explaining the data across subjects and conditions was Model 2 (a corticothalamic network with a common thalamic source) with a $92 \%$ posterior probability. Figure 3 shows individual fits between predicted and observed source-level power spectra and cross-spectral densities as computed for Model 2 by DCM.

To quantify changes in connectivity and excitability across conditions, we then examined the parameters of the best model (Model 2). Classical inference on the parameter estimates for each subject revealed a significant effect of condition on specific model parameters: one effect showed significant changes in thalamic excitability (Bonferroni corrected $p=0.01$ ), and the other a change in backward corticocortical connectivity from frontal to parietal cortices (Bonferroni corrected $p=0.036$ ) (Fig. 4, left). Post hoc analyses revealed that thalamic excitability significantly 


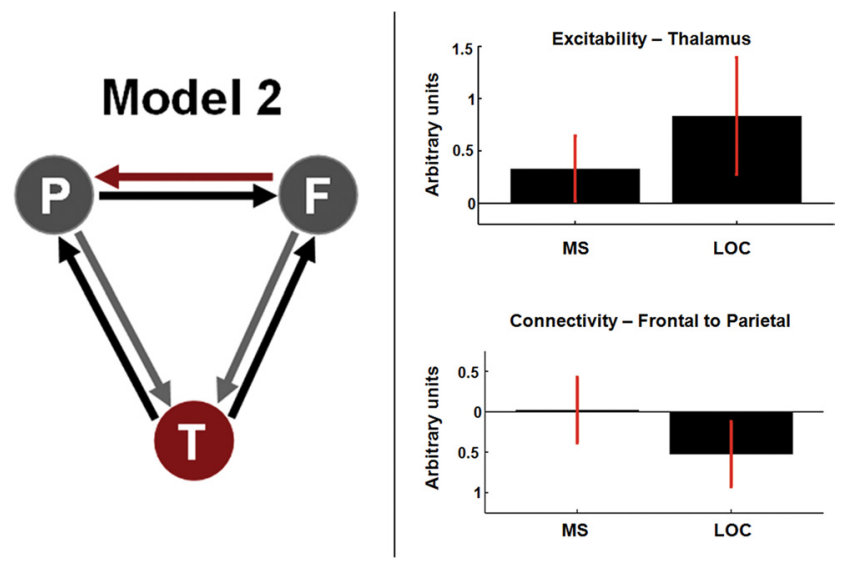

Figure 4. Connectivity parameter analysis of the winning model (i.e., Model 2, the model with a common thalamic source). Left, Quantitative parameter analysis revealed two effects of changes in conscious level on network parameters ( $p<0.05$, represented in dark red): changes in thalamic (T) excitability and changes in backward frontoparietal (F-P) connectivity. Right, Average change in thalamic excitability and frontoparietal backward connectivity parameters over subjects (with SEs shown as red bars) for mild sedation (MS) and LOC compared with wakefulness. Post hoc analyses revealed that changes in thalamic excitability were already present in mild sedation stage compared with wakefulness $(p<0.05)$, and persisted during LOC compared with wakefulness ( $p<0.05$ ), but without a significant further increase compared with mild sedation. In contrast, backward frontoparietal connectivity was not significantly modified during mild sedation, but showed a selective decrease $(p<0.05)$ during LOC compared with wakefulness. The (adimensional) changes are relative to the value in the first (waking) condition.

increased during mild sedation compared with wakefulness, and that this hyperexcitability persisted during loss of consciousness (Fig. 4, right). Hence, thalamic excitability mirrored the changes in fast rhythms that accompany propofol infusion but did not change with LOC. On the other hand, backward corticocortical connectivity was preserved during mild sedation, but showed a significant reduction with loss of consciousness - thus following the expression of slow power changes in EEG (Fig. 4, right). We observed a significant interaction between backward and forward connectivity changes with propofol-induced sedation stages $(p=$ $0.006)$. Thalamocortical connectivity and cortical excitability did not show significant changes across sedation stages. A complementary analysis of Model 1 (corticocortical model) parameters confirmed a reduction in backward connections during propofol-induced loss of consciousness (effect of the level of consciousness on backward connectivity: $p=0.022$ ). We also reproduced the significant interaction between backward and forward connectivity changes during propofol sedation $(p=0.026)$ under this model.

\section{Discussion}

To the extent that the models of thalamocortical interactions examined here are representative, DCM indicates that propofol-induced LOC is associated with a selective breakdown of corticocortical backward connectivity, while thalamocortical connectivity, thalamic excitability, and cortical excitability are preserved. These results extend previous studies reporting decreased cerebral connectivity during anesthesia-induced LOC using resting state fMRI (Boveroux et al., 2010; Martuzzi et al., 2010) or transcranial magnetic stimulationEEG (Ferrarelli et al., 2010). They suggest that propofol-induced LOC is due to impairment of direct cortical interactions, rather than to a thalamic inhibiting effect (Alkire et al., 2008; Franks, 2008). There are several issues that previous studies of propofol and functional connectivity could not resolve. Previous resting-state fMRI studies could not differentiate between impaired thalamocortical or corticocortical connectivity, or between an impairment of backward versus forward connections (Boveroux et al., 2010). Previous EEG functional connectivity studies (Cimenser et al., 2011; Murphy et al., 2011; Supp et al., 2011) provided contradictory results, suggesting increased coherence during propofol-induced LOC. The present study resolves these issues, through the analysis of directed effective connectivity using DCM. Our results support the view that propofol-induced LOC may be linked to an impairment of direct communication between cortical areas. This selective cortical effect is in line with previous studies reporting aroused thalamic EEG activity, but marked cortical slowing during propofol-induced LOC (Velly et al., 2007) or spike-wave seizures (Englot et al., 2009). Since bilateral thalamic lesions can cause a vegetative state (Adams et al., 2000), thalamic activity might be necessary, but not sufficient, for consciousness. The common denominator in the neural correlates of consciousness might rather be changes in distributed corticocortical processing.

According to the DCM conducted here, during propofolinduced LOC, backward corticocortical connectivity was impaired, while forward connectivity was preserved. Our findings do not point to a global decrease in connectivity. The significant interaction between backward and forward corticocortical connectivity changes during propofol-induced LOC rather suggests a selective impairment of backward connections, in the context of preserved forward connectivity. Our results are in line with previous scalp EEG studies reporting loss of front-to-back connectivity during propofol-induced sedation (Lee et al., 2009; Ku et al., 2011). The present finding supports a role for recurrent processing in higher-order cortices in conscious perception (Lamme, 2006; Boly et al., 2011). It is probably the case that both forward and backward connections are important for consciousness (Boly et al., 2011). Furthermore, the present results do not preclude more widespread physiological changes under other anesthetics. Rather, they suggest that backward connectivity in higher-order associative cortex may be a critical bottleneck for anomalies of conscious awareness.

Increases in the power of high frequencies, present from the start of propofol infusion, appeared to be linked to thalamic hyperexcitability, broadcasting abnormal rhythms to the cortex. According to DCM, thalamic excitability increased during both sedation and LOC compared with wakefulness. BMS suggested that a thalamic source interconnected with both cortical regions was the best model to explain our data. If thalamic involvement was restricted to specific nuclei (such as the pulvinar or geniculate, mainly connected to posterior cortex, or the anterior thalamus, mainly connected to anterior cortex), Model 3 would have won in BMS. DCM allows a principled comparison of different models (Penny et al., 2004), where the best model is not necessarily the most complex: in our case, the second model had greater evidence despite the fact it was simpler (had fewer free parameters) than the third model. Some studies show interconnections among PCC, ACC, and associative thalamic nuclei in humans (e.g., the centromedial thalamus) (Eckert et al., 2011). In monkeys, calbindin-positive matrix cells, concentrated within intralaminar thalamic nuclei, project diffusely to superficial layers of cortex (Jones, 2002). Cells in intralaminar nuclei can fire at high frequencies (Alkire et al., 2008). Thalamic hyperexcitability is in line with reports showing that propofol increases the discharge probability of thalamic relay cells (Angel and LeBeau, 1992; Bonhomme et al., 2001) and with a recent modeling study suggesting that increased thalamocortical coherence could explain propofol-induced changes in power spectra, especially in 
the alpha range (Ching et al., 2010). The present study highlights how DCM can separate mixed power spectrum patterns and provide a biophysically grounded explanation for them. Here, the two different phenomena expressed in the changes of power spectra (in the low and high frequencies) were related to two distinct neuronal mechanisms.

Several technical issues of the present work deserve discussion. DCM is a hypothesis-driven method: it does not explore all possible models but tests specific mechanistic hypotheses (Boly et al., 2011). Results of DCM studies are only valid within the space of the models tested. Our three models correspond to three hypotheses about generic changes in brain connectivity during propofol-induced LOC. The presence of high delta power in the EEG may reflect the expression of neuronal up and down states at smaller temporal and spatial scales; DCM for SSR, however, focuses on population dynamics under steady-state assumptions (i.e., condition-specific changes in power spectra) (Moran et al., 2009). State-dependent coupling in DCM is modeled using nonlinearities, which can be thought of as mediating activitydependent changes in synaptic efficacy. In DCM for SSRs, these nonlinearities are fairly simple and reduce to a sigmoid activation function for each neuronal subpopulation (Moran et al., 2009). In the present case, this model captures most changes in power present in source-level EEG (Fig. 3). The implicit mesoscopic characterization of synaptic responses (Moran et al., 2007, 2009) provided a reasonable account of electrophysiological data in a number of validation studies in humans or animals (Moran et al., 2008, 2011a,b).

We used source-reconstructed EEG responses, obtained using an L2-norm algorithm (Murphy et al., 2009), to access frontal and parietal signals under the different anesthetic conditions. The present study thus does not attempt to fully account for scalp EEG changes during propofol sedation, but rather accounts for selective changes in a frontoparietal network (encompassing ACC and PCC regions) that may represent propofol-induced changes in thalamocortical interactions. However, we cannot exclude the possibility that other networks may have shown different changes during propofol sedation. Our rationale was to choose two regions with demonstrable differences in power between wakefulness and propofol-induced LOC-here the ACC and PCC - to model with DCM. Furthermore, numerous studies have implicated the default network (Boly et al., 2009; Boveroux et al., 2010; Martuzzi et al., 2010; Vanhaudenhuyse et al., 2010; Sämann et al., 2011) and PCC (Laureys et al., 2004; Vogt and Laureys, 2005; Boveroux et al., 2008; Vanhaudenhuyse et al., 2010) in consciousness. Finally, the choice of ACC and PCC allowed us to compare our DCM results to the coherence analyses reported in (Murphy et al., 2011). As the source-reconstructed EEG signal-to-noise ratio decreases with source depth (Goldenholz et al., 2009), we chose to model a thalamic third driver as a hidden source in our analysis, rather than to extract the thalamic signal itself - an approach previously used in a number of EEG computational modeling studies (Breakspear et al., 2006; Ching et al., 2010; Victor et al., 2011). The architecture of this hidden source was chosen to model reticular and relay nuclei in the thalamus (Robinson et al., 2001; Breakspear et al., 2006). Without actual data from thalamus, we can only infer the presence of a thalamic relay. However, it is possible that this hidden source could reflect other brainstem contributions. Having said this, cortical-basal ganglia loops outputs involve the thalamus (Herrero et al., 2002; Redgrave et al., 2010)—in this context, a modulatory effect mediated by basal ganglia would be accounted for by a change in corticothalamic interactions our model. Our hy- pothesis that the thalamus was involved in propofol-induced changes is motivated by several studies suggesting a role for the thalamus in anesthesia-induced LOC (Alkire and Miller, 2005; Alkire et al., 2008). It also appeals to previous studies using a thalamocortical model to explain changes in EEG in pathological (Victor et al., 2011) or pharmacological (Ching et al., 2010) LOC. Further work in animals (Imas et al., 2005a,b) may provide direct thalamic measurements to corroborate the present findings.

Beyond the implications for the mechanisms underlying propofol-induced sedation, our results may provide further evidence for a key role of backward connections in the maintenance of consciousness. Backward connections seem to be impaired in the vegetative state (Boly et al., 2011) and under isoflurane sedation (Imas et al., 2006). They are also thought to play a role in normal conscious access (Fahrenfort et al., 2007). The present study suggests that loss of consciousness may be induced by a selective impairment of corticocortical backward connectivity, even in the presence of preserved subcortical inputs to the cortex.

In conclusion, our results show that a breakdown of recurrent cortical processing can lead to propofol-induced LOC, even in the presence of preserved thalamus arousal and excitability. The present findings support the hypothesis of a direct cortical effect of propofol anesthetic. In the future, corticocortical connectivity measures could be tested as a clinical measure for the depth of propofol sedation.

\section{References}

Adams JH, Graham DI, Jennett B (2000) The neuropathology of the vegetative state after an acute brain insult. Brain 123:1327-1338.

Alkire MT, Miller J (2005) General anesthesia and the neural correlates of consciousness. Prog Brain Res 150:229-244.

Alkire MT, Haier RJ, Fallon JH (2000) Toward a unified theory of narcosis: brain imaging evidence for a thalamocortical switch as the neurophysiologic basis of anesthetic-induced unconsciousness. Conscious Cogn 9:370-386.

Alkire MT, Hudetz AG, Tononi G (2008) Consciousness and anesthesia. Science 322:876-880.

Angel A (1993) Central neuronal pathways and the process of anaesthesia. Br J Anaesth 71:148-163.

Angel A, LeBeau F (1992) A comparison of the effects of propofol with other anaesthetic agents on the centripetal transmission of sensory information. Gen Pharmacol 23:945-963.

Boly M, Tshibanda L, Vanhaudenhuyse A, Noirhomme Q, Schnakers C, Ledoux D, Boveroux P, Garweg C, Lambermont B, Phillips C, Luxen A, Moonen G, Bassetti C, Maquet P, Laureys S (2009) Functional connectivity in the default network during resting state is preserved in a vegetative but not in a brain dead patient. Hum Brain Mapp 30:2393-2400.

Boly M, Garrido MI, Gosseries O, Bruno MA, Boveroux P, Schnakers C, Massimini M, Litvak V, Laureys S, Friston K (2011) Preserved Feedforward but Impaired Top-down Processes in the Vegetative State. Science 332:858-862.

Bonhomme V, Fiset P, Meuret P, Backman S, Plourde G, Paus T, Bushnell MC, Evans AC (2001) Propofol anesthesia and cerebral blood flow changes elicited by vibrotactile stimulation: a positron emission tomography study. J Neurophysiol 85:1299-1308.

Boveroux P, Bonhomme V, Boly M, Vanhaudenhuyse A, Maquet P, Laureys S (2008) Brain function in physiologically, pharmacologically, and pathologically altered states of consciousness. Int Anesthesiol Clin 46:131-146.

Boveroux P, Vanhaudenhuyse A, Bruno MA, Noirhomme Q, Lauwick S, Luxen A, Degueldre C, Plenevaux A, Schnakers C, Phillips C, Brichant JF, Bonhomme V, Maquet P, Greicius MD, Laureys S, Boly M (2010) Breakdown of within- and between-network resting state functional magnetic resonance imaging connectivity during propofol-induced loss of consciousness. Anesthesiology 113:1038-1053.

Breakspear M, Roberts JA, Terry JR, Rodrigues S, Mahant N, Robinson PA (2006) A unifying explanation of primary generalized seizures through nonlinear brain modeling and bifurcation analysis. Cereb Cortex 16:1296-1313. 
Ching S, Cimenser A, Purdon PL, Brown EN, Kopell NJ (2010) Thalamocortical model for a propofol-induced alpha-rhythm associated with loss of consciousness. Proc Natl Acad Sci U S A 107:22665-22670.

Cimenser A, Purdon PL, Pierce ET, Walsh JL, Salazar-Gomez AF, Harrell PG, Tavares-Stoeckel C, Habeeb K, Brown EN (2011) Tracking brain states under general anesthesia by using global coherence analysis. Proc Natl Acad Sci U S A 108:8832-8837.

David O, Friston KJ (2003) A neural mass model for MEG/EEG: coupling and neuronal dynamics. Neuroimage 20:1743-1755.

David O, Harrison L, Friston KJ (2005) Modelling event-related responses in the brain. Neuroimage 25:756-770.

Eckert U, Metzger CD, Buchmann JE, Kaufmann J, Osoba A, Li M, Safron A, Liao W, Steiner J, Bogerts B, Walter M (2011) Preferential networks of the mediodorsal nucleus and centromedian-parafascicular complex of the thalamus-A DTI tractography study. Hum Brain Mapp. Advance online publication. Retrieved April 15, 2012. doi:10.1002/hbm.21389.

Englot DJ, Modi B, Mishra AM, DeSalvo M, Hyder F, Blumenfeld H (2009) Cortical deactivation induced by subcortical network dysfunction in limbic seizures. J Neurosci 29:13006-13018.

Fahrenfort JJ, Scholte HS, Lamme VA (2007) Masking disrupts reentrant processing in human visual cortex. J Cogn Neurosci 19:1488-1497.

Felleman DJ, Van Essen DC (1991) Distributed hierarchical processing in the primate cerebral cortex. Cereb Cortex 1:1-47.

Ferrarelli F, Massimini M, Sarasso S, Casali A, Riedner BA, Angelini G, Tononi G, Pearce RA (2010) Breakdown in cortical effective connectivity during midazolam-induced loss of consciousness. Proc Natl Acad Sci U S A 107:2681-2686.

Fiset P, Paus T, Daloze T, Plourde G, Meuret P, Bonhomme V, Hajj-Ali N, Backman SB, Evans AC (1999) Brain mechanisms of propofol-induced loss of consciousness in humans: a positron emission tomographic study. J Neurosci 19:5506-5513.

Franks NP (2008) General anaesthesia: from molecular targets to neuronal pathways of sleep and arousal. Nat Rev Neurosci 9:370-386.

Friston KJ, Stephan KE (2007) Free-energy and the brain. Synthese 159:417-458.

Friston KJ, Harrison L, Penny W (2003) Dynamic causal modelling. Neuroimage 19:1273-1302.

Friston K, Mattout J, Trujillo-Barreto N, Ashburner J, Penny W (2007) Variational free energy and the Laplace approximation. Neuroimage 34:220-234.

Goldenholz DM, Ahlfors SP, Hämäläinen MS, Sharon D, Ishitobi M, Vaina LM, Stufflebeam SM (2009) Mapping the signal-to-noise-ratios of cortical sources in magnetoencephalography and electroencephalography. Hum Brain Mapp 30:1077-1086.

Grave de Peralta Menendez R, Gonzalez Andino S, Lantz G, Michel CM, Landis T (2001) Noninvasive localization of electromagnetic epileptic activity. I. Method descriptions and simulations. Brain Topogr 14:131-137.

Herrero MT, Barcia C, Navarro JM (2002) Functional anatomy of thalamus and basal ganglia. Childs Nerv Syst 18:386-404.

Imas OA, Ropella KM, Ward BD, Wood JD, Hudetz AG (2005a) Volatile anesthetics disrupt frontal-posterior recurrent information transfer at gamma frequencies in rat. Neurosci Lett 387:145-150.

Imas OA, Ropella KM, Ward BD, Wood JD, Hudetz AG (2005b) Volatile anesthetics enhance flash-induced gamma oscillations in rat visual cortex. Anesthesiology 102:937-947.

Imas OA, Ropella KM, Wood JD, Hudetz AG (2006) Isoflurane disrupts anterio-posterior phase synchronization of flash-induced field potentials in the rat. Neurosci Lett 402:216-221.

Jacobson S, Trojanowski JQ (1977) Prefrontal granular cortex of the rhesus monkey. I. Intrahemispheric cortical afferents. Brain Res 132:209-233.

Jansen BH, Rit VG (1995) Electroencephalogram and visual evoked potential generation in a mathematical model of coupled cortical columns. Biol Cybern 73:357-366.

Jones EG (2002) Thalamic circuitry and thalamocortical synchrony. Philos Trans R Soc Lond B Biol Sci 357:1659-1673.

Kiebel SJ, Garrido MI, Friston KJ (2007) Dynamic causal modelling of evoked responses: the role of intrinsic connections. Neuroimage 36:332-345.

Kiebel SJ, Garrido MI, Moran R, Chen CC, Friston KJ (2009) Dynamic causal modeling for EEG and MEG. Hum Brain Mapp 30:1866-1876.
Kirschstein T, Köhling R (2009) What is the source of the EEG? Clin EEG Neurosci 40:146-149.

Ku SW, Lee U, Noh GJ, Jun IG, Mashour GA (2011) Preferential inhibition of frontal-to-parietal feedback connectivity is a neurophysiologic correlate of general anesthesia in surgical patients. PLoS One 6:e25155.

Lamme VA (2006) Towards a true neural stance on consciousness. Trends Cogn Sci 10:494-501.

Laureys S, Owen AM, Schiff ND (2004) Brain function in coma, vegetative state, and related disorders. Lancet Neurol 3:537-546.

Lee U, Kim S, Noh GJ, Choi BM, Hwang E, Mashour GA (2009) The directionality and functional organization of frontoparietal connectivity during consciousness and anesthesia in humans. Conscious Cogn 18: 1069-1078.

Llinás R, Ribary U, Contreras D, Pedroarena C (1998) The neuronal basis for consciousness. Philos Trans R Soc Lond B Biol Sci 353:1841-1849.

Marsh B, White M, Morton N, Kenny GN (1991) Pharmacokinetic model driven infusion of propofol in children. Br J Anaesth 67:41-48.

Martuzzi R, Ramani R, Qiu M, Rajeevan N, Constable RT (2010) Functional connectivity and alterations in baseline brain state in humans. Neuroimage 49:823-834.

Moran RJ, Kiebel SJ, Stephan KE, Reilly RB, Daunizeau J, Friston KJ (2007) A neural mass model of spectral responses in electrophysiology. Neuroimage 37:706-720.

Moran RJ, Stephan KE, Kiebel SJ, Rombach N, O’Connor WT, Murphy KJ, Reilly RB, Friston KJ (2008) Bayesian estimation of synaptic physiology from the spectral responses of neural masses. Neuroimage 42:272-284.

Moran RJ, Stephan KE, Seidenbecher T, Pape HC, Dolan RJ, Friston KJ (2009) Dynamic causal models of steady-state responses. Neuroimage 44:796-811.

Moran RJ, Symmonds M, Stephan KE, Friston KJ, Dolan RJ (2011a) An in vivo assay of synaptic function mediating human cognition. Curr Biol 21:1320-1325.

Moran RJ, Mallet N, Litvak V, Dolan RJ, Magill PJ, Friston KJ, Brown P (2011b) Alterations in brain connectivity underlying beta oscillations in Parkinsonism. PLoS Comput Biol 7:e1002124.

Murphy M, Riedner BA, Huber R, Massimini M, Ferrarelli F, Tononi G (2009) Source modeling sleep slow waves. Proc Natl Acad Sci U S A 106:1608-1613.

Murphy M, Bruno MA, Riedner BA, Boveroux P, Noirhomme Q, Landsness EC, Brichant JF, Phillips C, Massimini M, Laureys S, Tononi G, Boly M (2011) Propofol anesthesia and sleep: a high-density EEG study. Sleep 34:283-291A.

Nakakimura K, Sakabe T, Funatsu N, Maekawa T, Takeshita H (1988) Metabolic activation of intercortical and corticothalamic pathways during enflurane anesthesia in rats. Anesthesiology 68:777-782.

Nichols TE, Holmes AP (2002) Nonparametric permutation tests for functional neuroimaging: a primer with examples. Hum Brain Mapp 15:1-25.

Pandya DN (1995) Anatomy of the auditory cortex. Rev Neurol (Paris) 151:486-494.

Penny WD, Stephan KE, Mechelli A, Friston KJ (2004) Comparing dynamic causal models. Neuroimage 22:1157-1172.

Ramsay MA, Savege TM, Simpson BR, Goodwin R (1974) Controlled sedation with alphaxalone-alphadolone. Br Med J 2:656-659.

Redgrave P, Rodriguez M, Smith Y, Rodriguez-Oroz MC, Lehericy S, Bergman H, Agid Y, DeLong MR, Obeso JA (2010) Goal-directed and habitual control in the basal ganglia: implications for Parkinson's disease. Nat Rev Neurosci 11:760-772.

Robinson PA, Rennie CJ, Wright JJ, Bahramali H, Gordon E, Rowe DL (2001) Prediction of electroencephalographic spectra from neurophysiology. Phys Rev E Stat Nonlin Soft Matter Phys 63:021903.

Rodrigues S, Chizhov AV, Marten F, Terry JR (2010) Mappings between a macroscopic neural-mass model and a reduced conductance-based model. Biol Cybern 102:361-371.

Rouiller EM, Durif C (2004) The dual pattern of corticothalamic projection of the primary auditory cortex in macaque monkey. Neurosci Lett 358:49-52.

Sämann PG, Wehrle R, Hoehn D, Spoormaker VI, Peters H, Tully C, Holsboer F, Czisch M (2011) Development of the brain's default mode network from wakefulness to slow wave sleep. Cereb Cortex 21:2082-2093.

Schiff ND, Plum F (2000) The role of arousal and "gating" systems in the neurology of impaired consciousness. J Clin Neurophysiol 17:438-452.

Schwartz ML, Goldman-Rakic PS (1984) Callosal and intrahemispheric 
connectivity of the prefrontal association cortex in rhesus monkey: relation between intraparietal and principal sulcal cortex. J Comp Neurol 226:403-420.

Stephan KE, Penny WD, Daunizeau J, Moran RJ, Friston KJ (2009) Bayesian model selection for group studies. Neuroimage 46:1004-1017.

Steriade M (2006) Grouping of brain rhythms in corticothalamic systems. Neuroscience 137:1087-1106.

Supp GG, Siegel M, Hipp JF, Engel AK (2011) Cortical hypersynchrony predicts breakdown of sensory processing during loss of consciousness. Curr Biol 21:1988-1993.

Vahle-Hinz C, Detsch O, Siemers M, Kochs E (2007) Contributions of GABAergic and glutamatergic mechanisms to isoflurane-induced suppression of thalamic somatosensory information transfer. Exp Brain Res 176:159-172.

Vanhaudenhuyse A, Noirhomme Q, Tshibanda LJ, Bruno MA, Boveroux P, Schnakers C, Soddu A, Perlbarg V, Ledoux D, Brichant JF, Moonen G, Maquet P, Greicius MD, Laureys S, Boly M (2010) Default network con- nectivity reflects the level of consciousness in non-communicative braindamaged patients. Brain 133:161-171.

Velly LJ, Rey MF, Bruder NJ, Gouvitsos FA, Witjas T, Regis JM, Peragut JC, Gouin FM (2007) Differential dynamic of action on cortical and subcortical structures of anesthetic agents during induction of anesthesia. Anesthesiology 107:202-212.

Victor JD, Drover JD, Conte MM, Schiff ND (2011b) Mean-field modeling of thalamocortical dynamics and a model-driven approach to EEG analysis. Proc Natl Acad Sci U S A 108 [Suppl 3]:15631-15638.

Vogt BA, Laureys S (2005) Posterior cingulate, precuneal and retrosplenial cortices: cytology and components of the neural network correlates of consciousness. Prog Brain Res 150:205-217.

Winguth SD, Winer JA (1986) Corticocortical connections of cat primary auditory cortex (AI): laminar organization and identification of supragranular neurons projecting to area AII. J Comp Neurol 248: $36-56$. 